\title{
Holography abhors visible trapped surfaces
}

\section{Netta Engelhardt and Åsmund Folkestad}

Center for Theoretical Physics, Massachusetts Institute of Technology, Cambridge, MA 02139, U.S.A.

E-mail: engeln@mit.edu, afolkest@mit.edu

ABSTRACT: We prove that consistency of the holographic dictionary implies a hallmark prediction of the weak cosmic censorship conjecture: that in classical gravity, trapped surfaces lie behind event horizons. In particular, the existence of a trapped surface implies the existence of an event horizon, and that furthermore this event horizon must be outside of the trapped surface. More precisely, we show that the formation of event horizons outside of a strong gravity region is a direct consequence of causal wedge inclusion, which is required by entanglement wedge reconstruction. We make few assumptions beyond the absence of evaporating singularities in strictly classical gravity. We comment on the implication that spacetimes with naked trapped surfaces do not admit a holographic dual, note a possible application to holographic complexity, and speculate on the dual CFT interpretation of a trapped surface.

KEYwords: AdS-CFT Correspondence, Black Holes, Spacetime Singularities

ArXiv EPrint: 2012.11445 


\section{Contents}

1 Introduction $\quad 1$

1.1 Assumptions and conventions 5

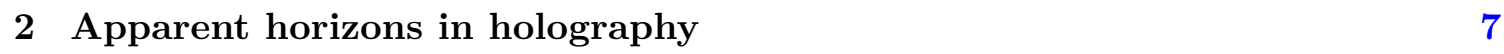

3 Apparent horizons lie behind event horizons $\quad 9$

4 Trapped surfaces lie behind event horizons $r$

$\begin{array}{lll}5 & \text { Discussion } & 17\end{array}$

\section{Introduction}

Foundational results in modern gravitational physics, from black hole thermodynamics [13] to topological censorship [4], often rely on the absence of strong gravity outside of horizons, or more precisely the weak cosmic censorship conjecture [5]. Gedankenexperiments that rely upon the formation of black holes from generic matter collapse require an implicit assumption that matter typically coalesces into black holes and not into naked singularities.

This assumption appears reasonable given the apparent absence of naked singularities in observational data to date. The more precise statement of weak cosmic censorship (which we shall henceforth refer to simply as "cosmic censorship") is the conjecture that the Einstein equation evolves generic regular initial data with certain asymptotics to a complete asymptotic infinity $\mathscr{I}[6,7]$. In its initial formulation it applied specifically to asymptotically flat initial data in four dimensions [5], but it has been generalized to other dimensions and asymptotics when a suitable asymptotic infinity $\mathscr{I}$ exists (see e.g. [8]).

Developments over the past decade have eroded confidence in the validity of this statement at least in its more general form: counterexamples have been found in asymptotically $\mathrm{AdS}_{4}$ [9-13] and in higher dimensional asymptotically flat space [14-21]. Even in fourdimensional asymptotically flat space, non-generic initial data can evolve to form naked singularities [22-24]. While it is in principle possible that cosmic censorship is in fact correct in four-dimensional asymptotically flat space (and for generic initial data), numerous extant violations in other settings suggest otherwise. Since results that rely on cosmic censorship are expected to be applicable in broad generality in arbitrary dimensions and often for AdS and dS asymptotics, violations of cosmic censorship - generic or otherwise - are problematic in any setting where classical gravity is expected to be valid.

It would be particularly unfortunate ${ }^{1}$ if such violations were to indicate that trapped surfaces can lie outside of event horizons. In its current formulation, cosmic censorship

\footnotetext{
${ }^{1}$ Or fortunate, from a certain point of view.
} 
forbids the existence of trapped surfaces - i.e. surfaces from which light rays converge in any direction due to gravitational lensing — outside of event horizons [25]. A large set of theorems in classical gravity relies on this result (see [25, 26]). Numerical relativity typically uses the detection of (marginally) trapped surfaces as an avatar for the event horizon, whose location (and existence) can only be determined in infinite time. Must we face the possibility that these results are all questionable?

Possibly not, at least in spacetimes that arise as classical limits of quantum gravity. The recent discoveries of violations of cosmic censorship in $\mathrm{AdS}_{4}$ [9-13] have also been found to violate the Weak Gravity Conjecture [27]; cosmic censorship is restored precisely when the theory is adjusted so as to satisfy this conjecture, which is hypothesized to discriminate between spacetimes that do and do not admit valid UV completions. The confluence of validity of the cosmic censorship and the weak gravity conjecture has given rise to speculation that while classical General Relativity admits violations of cosmic censorship, the classical spacetimes that result from a truncation of a valid quantum theory of gravity do not $[11,13]$ : that is, that quantum gravity enforces cosmic censorship on its strict classical limit.

Here we focus on trapped surfaces rather than the statement of cosmic censorship in terms of initial data. The restriction of trapped surfaces to lie behind horizons is one of the most valuable consequences of cosmic censorship, since as noted above, it is a sine qua non for a number of results in General Relativity [26]. It is furthermore one of relatively few consequences of cosmic censorship that can be formulated in terms of the experience of a family of observers: a trapped surface outside of a horizon can in principle be detected by an asymptotic family of observers in finite time (or retarded time, in the asymptotically flat case). In fact, one could even go so far as to argue that the absence of trapped surfaces outside of horizons is a large part of the physical content of the weak cosmic censorship conjecture; namely that regions of strong gravity (usually heralded by trapped surfaces) are hidden from asymptotic observers. This statement also fortunately avoids any references to singularities, which are notoriously hard to work with — see [28-31] and references therein for literature in General Relativity attempting classify strengths and types of singularities. ${ }^{2}$

We aim to test the hypothesis that quantum gravity forces trapped surfaces behind horizons: we use holography as a laboratory for the classical limit of quantum gravity and ask whether some principle of the AdS/CFT correspondence implies that trapped surfaces remain cloaked from the asymptotic boundary.

Concrete evidence in favor of such a conclusion was found recently in e.g. [33], which used the holographic dictionary to prove the Penrose Inequality in AdS [34-36], a key result implied by the combination of two oft-quoted but unproven conjectures: (1) that trapped surfaces lie behind horizons, and (2) that black holes equilibrate. The proof of [33] assumed neither (1) nor (2) but instead made use of the holographic entanglement entropy proposal of Ryu-Takayanagi [37] and Hubeny-Rangamani-Takayanagi [38] (HRT)

$$
S_{\mathrm{vN}}\left[\rho_{R}\right]=\frac{\operatorname{Area}\left[X_{R}\right]}{4 G \hbar},
$$

\footnotetext{
${ }^{2}$ For instance, as discussed in [32], a singularity like the Gregory-Laflamme instability would by any nice definition be considered "weak" enough to be allowed by cosmic censorship. We thank R. Emparan for discussions on this topic.
} 
where $\rho_{R}$ is the density matrix of the CFT state reduced to the region $R$ and $X_{R}$ is the minimal area stationary surface homologous to $R$.

Because the Penrose Inequality follows from the absence of trapped surfaces outside of horizons together with black hole equilibration, it is a good omen in favor of cosmic censorship; however, it falls well short of proving that trapped surfaces in fact must lie behind horizons. ${ }^{3}$

In this article, we close this gap, thus proving a central consequence of cosmic censorship: the holographic dictionary implies that trapped surfaces lie behind event horizons. Our primary assumptions ${ }^{4}$ are (1) the HRT prescription, (2) that there exist unitary operators on the boundary whose effect in the bulk propagates causally, and (3) that singularities do not evaporate (a criterion that will be defined more rigorously in the following section) in classical gravity without violations of the null energy condition. Steps (1) and (2) together imply causal wedge inclusion [39, 40]: that the causal wedge must lie inside of the entanglement wedge. This holographic ingredient is a key step in our proof.

Before proceeding to the outline of the proof, let us briefly comment on quantum corrections. When quantum gravity effects are taken into account and violations of the null energy condition $\left(T_{a b} k^{a} k^{b} \geq 0\right.$ for null $\left.k^{a}\right)$ are permitted, there is of course no expectation that the above formulation of cosmic censorship should remain valid; it is, after all, a prediction about the behavior of classical general relativity. Indeed, evaporating black holes do have trapped surfaces outside of horizons (see e.g. [41]). However, cosmic censorship is a statement about the classical theory; we are primarily concerned with ascertaining whether the classical limit of quantum gravity features trapped surfaces outside of event horizons. Within semiclassical gravity, however, these results may be interpreted as statements about early stages of gravitational collapse.

Outline of the proof: the proof has three ingredients: first, the HRT prescription (1.1) combined with the fact that turning on local unitary operators in the dual theory at $\mathscr{I}$ results in causally propagating bulk perturbations; second, a theorem of [42] proving that an apparent horizon must lie between trapped surfaces and "normal" surfaces - surfaces in which ingoing light rays converge and outgoing light rays expand; and third, the holographic description of apparent horizons [43,44] (which is not an additional ingredient but rather a construction that relies only on HRT).

First, we use the fact that $S_{\mathrm{vN}}[\rho]$ is invariant under local unitary operations on the boundary state $\rho$ to argue that the HRT surface $X$ of a connected component of $\mathscr{I}$ cannot be timelike separated to any portion of $\mathscr{I}$. This is a well-established requirement often referred to as causal wedge inclusion $[39,40]$ : if $X$ were timelike-separated to $\mathscr{I}$, then it would be possible for a local unitary CFT operator acting to create a bulk signal propagating causally to $X$, which could modify its area; this is illustrated in figure 1 . Thus we

\footnotetext{
${ }^{3}$ And falls even shorter of proving cosmic censorship.

${ }^{4}$ We also make some technical assumptions on the past of these trapped surfaces (but not their future, which is the primary point of concern for cosmic censorship) and a genericity assumption. Both assumptions are manifestly true in spacetimes with spherical symmetry, and we motivate the more general assumptions in the body of the paper.
} 


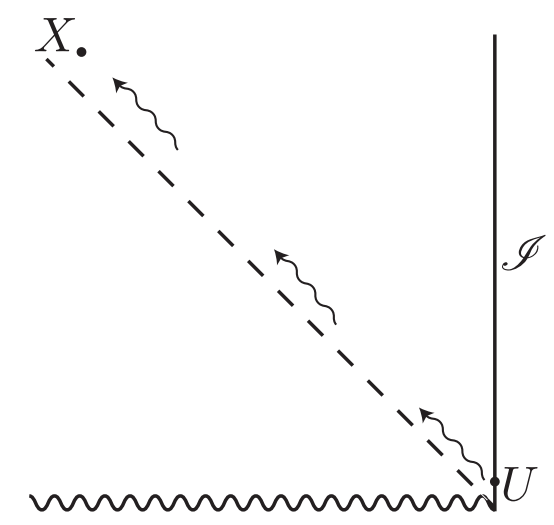

Figure 1. Example showing how causal connection between $\mathscr{I}$ and its HRT surface $X$ can be used to change $S_{\mathrm{vN}}$ with a local unitary.

would find that if $X$ were timelike to any $p \in \mathscr{I}$ it would be possible to modify $S_{\mathrm{vN}}[\rho]$ via local unitaries acting on $\rho .{ }^{5}$

Next, the theorem of [42] guarantees that a certain type of apparent horizon always lies between normal and trapped surfaces. We show that, given a trapped surface in our setup (including the assumptions of footnote 4), there are always normal surfaces outside of it, and that the type of apparent horizon guaranteed by the theorem satisfies the refinement necessary for step three.

Finally, we use the holographic construction of a dual to this type of apparent horizon $[43,44]$. The construction instructs us to fix the spacetime and matter outside of the apparent horizon - its so-called outer wedge - and modify the spacetime elsewhere via a specific prescription. If naked singularities and Cauchy horizons develop as a result of this prescription, we permit any extension beyond the Cauchy horizon consistent with our relatively mild assumptions about General Relativity (here we operate under the assumption that the boundary conditions are inherited by a top-down UV completion). In this newly constructed spacetime, the HRT surface $X$ is null-separated from the apparent horizon as illustrated in figure 2. Since by step one there are no timelike curves from the HRT surface to $\mathscr{I}$, there can be no future-directed timelike curves from the apparent horizon to $\mathscr{I}$ : we find that apparent horizons must lie outside of $I^{-}[\mathscr{I}]$ in the coarse-grained spacetime; under the assumption that singularities do not evaporate in classical spacetimes satisfying the null energy condition, we can then deduce that apparent horizons must therefore also lie behind the event horizon in the original spacetime. ${ }^{6}$ This immediately shows that

\footnotetext{
${ }^{5}$ Causal wedge inclusion can also be shown to follow from entanglement wedge nesting [45], which states that if the size of a CFT subregion increases from $R_{1}$ to $R_{2} \supset R_{1}$, the part of the bulk that could be reconstructed from $R_{1}$ can also be reconstructed from $R_{2}$.

${ }^{6}$ Since, to our knowledge, known solutions with evaporating singularities - a concept that we will make precise in section 3 - arising from the evolution of initial data feature violations of the null energy condition, we do not find this assumption to be particularly prohibitive. More generally, violations of weak cosmic censorship are normally concerned with the formation of singularities rather than their demise. This will be discussed at greater length in section 5 .
} 


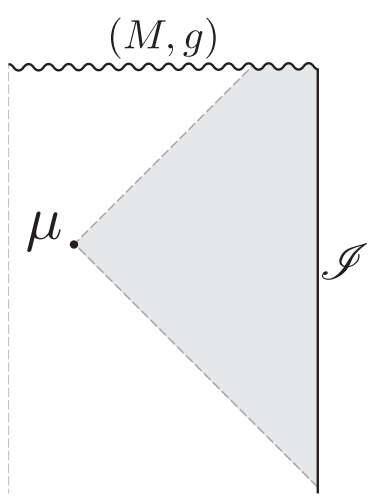

(a)

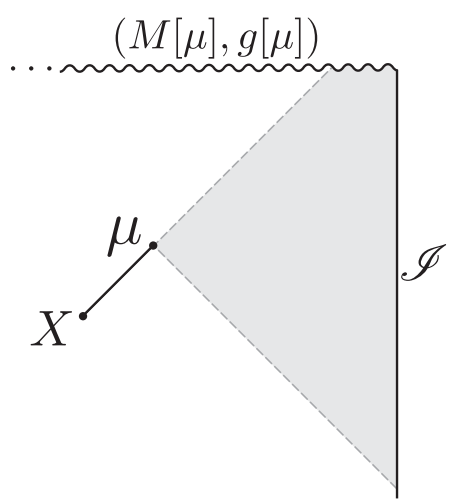

(b)

Figure 2. (a) An apparent horizon $\mu$ (more precisely, a minimar surface) in a spacetime $(M, g)$. (b) The same apparent horizon in its corresponding coarse-grained spacetime $(M[\mu], g[\mu])$, where there HRT surface $X$ with respect to $\mathscr{I}$ is null related to $\mu$. The shaded wedge outside $\mu$ is common to both spacetimes.

naked singularities - should they exist in holographic spacetimes - cannot incur trapped surfaces outside of horizons. The contrapositive then yields the following statement: if a given spacetime has a trapped surface outside of (or without a) horizon, it cannot be holographic; should we adopt the perspective that $\operatorname{AdS}_{D \geq 3}$ spacetimes with a well-defined UV completion are always holographic, this then becomes a potential swampland condition on the set of spacetimes with valid UV completions.

Relation to prior work: as alluded to earlier, previous attempts to prove various implications of the weak cosmic censorship conjecture have frequently encountered complications related to classifying singularities [28-31]. By considering trapped surfaces directly, and by defining evaporating singularities in terms of homology hypersurfaces (see Definition 1), we are able to avoid this complication altogether. Additionally, since cosmic censorship is known to be violated on a measure-zero set of the space of solutions to the Einstein equations [22-24], a genericity assumption is necessary for there to be any chance of the usual statement weak cosmic censorship to be true. The first half of our proof requires no assumptions about any genericity condition, and proves definitively that spacetimes with marginally trapped surfaces outside of horizons are not holographic. The second part of the proof, that all trapped surfaces are also behind horizons does assume a mild genericity condition and a technical assumption. We expect that these assumptions likely can be relaxed.

\subsection{Assumptions and conventions}

Assumptions: we will assume the null convergence condition, $R_{a b} k^{a} k^{b} \geq 0$ for all null vectors $k^{a}$ as well as the HRT prescription for computing $S_{\mathrm{vN}}$ (1.1) and entanglement wedge reconstruction. In particular, this means that we work strictly within classical General Relativity. We further assume that our spacetime is time-orientable and has no closed 


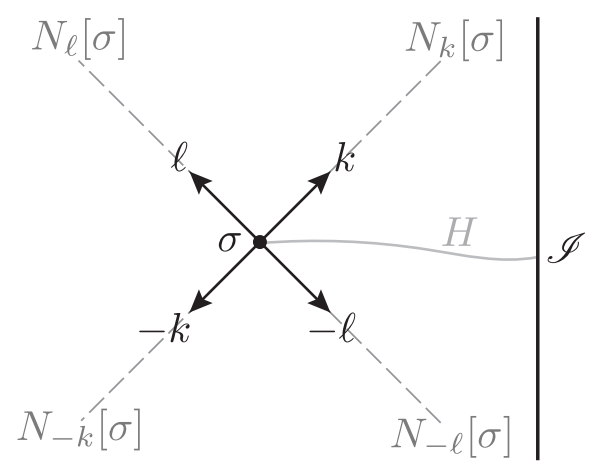

Figure 3. An illustration of the four null normals of a surface $\sigma$ (here shown to be homologous to $\mathscr{I})$ together with the corresponding null congruences.

timelike curves. We further assume that in a maximal conformal extension $(\partial M, h)$ of $\mathscr{I}$, $\mathscr{I}$ is both globally hyperbolic and geodesically complete; unless explicitly noted otherwise, by $\mathscr{I}$ we will always mean a connected component of $\partial M$. By contrast, $\partial M$ will always denote the complete conformal boundary. We assume that $\mathscr{I}$ is spatially compact.

Conventions: in describing domains of dependence that include the relevant portion of the asymptotic boundary, we will include $\mathscr{I}$ in any Cauchy slices of these domains of dependence, per standard conventions (see [46] for a definition).

1. Bulk: we assume that there are no closed causal curves and that $J^{+}(p) \cap J^{-}(q)$ is compact in the conformal completion for each pair of points $q, p$. This is typically referred to as the AdS version of global hyperbolicity; in a slight abuse of notation, we shall simply refer to it as global hyperbolicity. We refer to the past Cauchy horizon of $D$ as $\partial^{-} D$ and the future Cauchy horizon as $\partial^{+} D$. For a set $A \subset M$, the boundary of $A$ is denoted $\partial A$. We take $\hat{A}$ to be the closure of $A$ in the conformal completion $M \cup \partial M$. If an intersection between a subset of $M$ and $\mathscr{I}$ is taken, it is always implicitly assumed that the closure in the conformal completion is taken first. Examples of the sets described above are shown in figure 4 .

By a surface, we will always mean a spacelike (achronal) codimension-two embedded submanifold (without boundary). A surface $\sigma$ is said to be homologous to $\mathscr{I}$ if there is an achronal homology hypersurface $H$ between $\sigma$ and $C$; i.e. if there is an achronal hypersurface $H$ such that (1) $\partial H=\sigma \cup C$ where $C$ is a Cauchy slice of $\mathscr{I}$ and (2) $H$ is compact in the conformal completion of $(M, g)$. Any surface $\sigma$ (homologous to $\mathscr{I}$ or otherwise) has two linearly independent normals, which we may pick to be null. We will denote the future-directed null normals $k^{a}$ and $\ell^{a}$; if $\sigma$ is additionally homologous to $\mathscr{I}$, then at least one of these null normals points towards $\mathscr{I}$ - i.e. is "outwards-pointing"; we will name that vector $k^{a}{ }^{7}$ The four

\footnotetext{
${ }^{7}$ If $\partial M$ consists of multiple connected components $\mathscr{I}$ and $\sigma$ is homologous to multiple components, it is possible for both $k^{a}$ and $\ell^{a}$ to be outwards-pointing, and it will be either clear from context or immaterial which one is which.
} 
null geodesic congruences obtained by firing null geodesics along $\pm k^{a}$ and $\pm \ell^{a}$ from $\sigma$ and terminating at conjugate points and geodesic intersections are denoted $N_{ \pm k}[\sigma]$ and $N_{ \pm \ell}[\sigma]$. See figure 3 for an illustration of these concepts. We will generally be interested in the expansion of a null congruence in the $n^{a} \in\left\{\ell^{a}, k^{a}\right\}$ direction, defined as

$$
\theta_{n}=n^{a} \nabla_{a}(\ln \text { Area }[\sigma]) .
$$

A marginally trapped surface satisfies $\theta_{k}=0$ and $\theta_{\ell}<0$ (whereas if $\theta_{\ell}$ is unconstrained it is simply marginally outer trapped). A trapped surface has negative expansion along both future expansions.

Finally, as described in the introduction, we will work with what we shall refer to permissible extensions of a Cauchy horizon as an extension of a spacetime beyond a Cauchy horizon that is consistent with General Relativity and all our global assumptions listed above, together with one more condition: we assume that we never extend spacetimes beyond the "holographic region", should singularities terminating CFT evolution arise (see discussion below). All other conventions are as in [26].

2. Boundary: we use the letter $C$ to refer to Cauchy slices of any maximal conformal extension of $\mathscr{I}$, and by $i^{+}$we mean future timelike infinity of $M{ }^{8}$ Since the utility of our proof is in the provision of a UV complete description of the gravitating system via the CFT, we are also concerned with the evolution of the boundary theory. That is, if at any point the CFT (in the large- $N$ limit) becomes sick (e.g. if the stress tensor becomes divergent [47], Hamiltonian becomes unbounded from below, etc. [48]) in finite boundary time in a maximal conformal extension, we must conclude that this similarly puts an end to bulk evolution. Thus if the CFT evolution is well-defined (for $N \rightarrow \infty$ ) only between two (potentially empty) boundary time slices $C^{-}$and $C^{+}$of the maximal conformal extension $(\partial M, h), C^{+}$being to the future of $C^{-}$, then we excise $J^{+}\left[C^{+}\right]$and $J^{-}\left[C^{-}\right]$from $(M, g)$. This is because these regions are not encoded in the CFT state between $C^{-}$and $C^{+}$, and so the spacetime without the excision is not completely encoded in the CFT and thus not holographic. ${ }^{9}$ To facilitate terminology here, we define $\widetilde{i^{+}}$as the futuremost endpoint of the CFT evolution. If the CFT evolution exists for all time in the maximal conformal extension, then $i^{+}=\widetilde{i^{+}}$; otherwise, if the CFT evolution ends prematurely at some finite time, $\widetilde{i^{+}}$becomes the effective timelike infinity: any signals that travel from the bulk to the boundary and arrive in the future of $\widetilde{i^{+}}$are non-holographic.

\section{Apparent horizons in holography}

As discussed in section 1, an apparent horizon is intuitively the boundary to the very strong gravity region on a given spatial slice; more precisely, it is the boundary between normal

\footnotetext{
${ }^{8}$ Even though the commonly drawn conformal diagram of AdS does not show $i^{+}$, it nonetheless exists!

${ }^{9} \mathrm{An}$ example of a scenario like this is Choptuik critical collapse in AdS [47], where the CFT stress tensor becomes singular when the Cauchy horizon caused by the naked singularity reaches $\mathscr{I}$. Any potential extension of the spacetime to the future of this time is not holographically encoded in the CFT.
} 


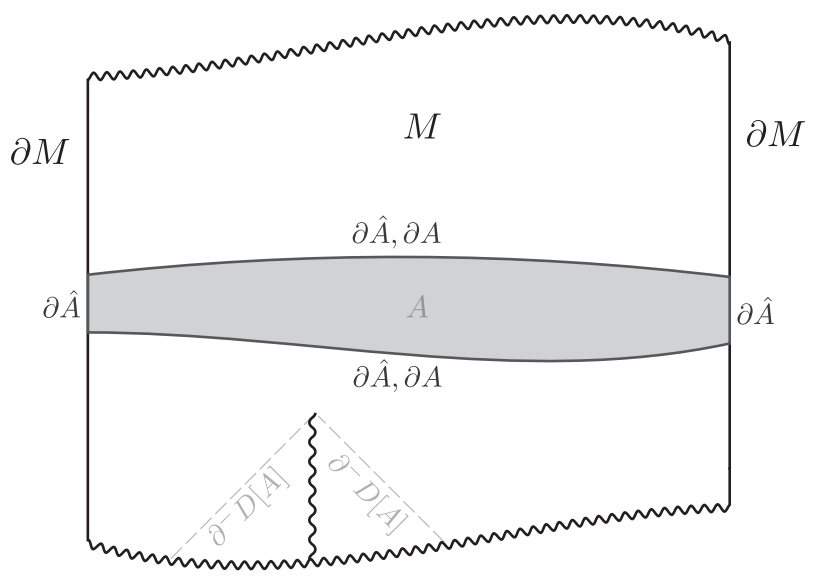

Figure 4. An example of an asymptotically AdS spacetime $M$ with conformal boundary $\partial M$, and with set $A \subset M$ marked. The boundaries of $A, \partial A$ in $M$ and $\partial \hat{A}$ in $M \cup \partial M$, are highlighted. Furthermore, the past Cauchy horizon of $D[A]=D[\partial A]$ is illustrated.

and trapped surfaces on a spatial slice. In this article we will be concerned with a slight refinement of apparent horizons called "minimar surfaces" [43].

A compact, connected surface $\mu$ is called a future minimar surface if it satisfies the following:

1. $\mu$ is marginally trapped;

2. $\mu$ is homologous to $\mathscr{I}$;

3. $\mu$ is strictly stable, meaning there exists choice of $k^{a}$ and $\ell^{a}$ such that $k^{a} \nabla_{a} \theta_{\ell}<0$ everywhere on $\mu$ (this effectively means that there exist untrapped surfaces outside of $\mu$ and trapped surfaces inside $\mu$ );

4. $\mu$ is the surface of least area on at least one of its homology hypersurfaces.

In analogy with the entanglement wedge, the "outer wedge" of $\mu$, denoted $O_{W}[\mu]$, is the domain of dependence of $H$. The absence of global hyperbolicity raises a potential concern that the outer wedge is dependent on the choice of homology hypersurface. In the subsequent section, we will prove that this concern is unfounded in the absence of evaporating singularities. Finally, note that it is possible to define a past analogue of a minimar by replacing $\ell \rightarrow-k, k \rightarrow-\ell$. All of our results will be valid for this time-reversed choice, but for brevity we will focus on future case. Consequently we often refer to future minimars just as minimars.

Our results rely heavily on the primary construction of the dual to the apparent horizon, which builds a spacetime in which the area of the HRT surface is identical to the area of $\mu$, and which agrees with $(M, g)$ on $O_{W}[\mu]$. The construction is illustrated and described in figure 5. Because the spacetime in question is generated by discarding the region that was originally behind $\mu$, it is often referred to as the coarse-grained spacetime, 


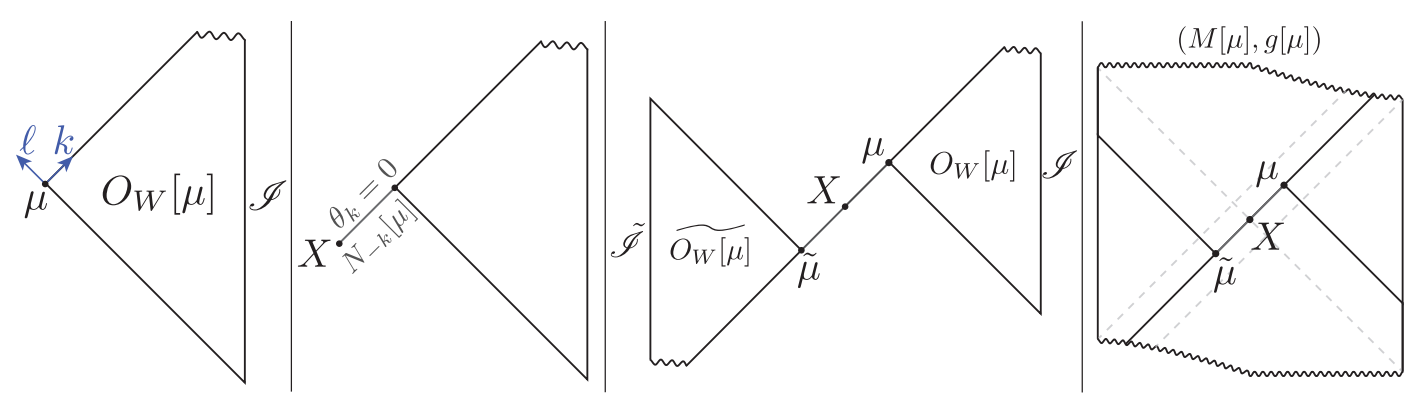

Figure 5. The coarse-grained spacetime construction of [43, 44]. The first panel shows the outer wedge of a minimar $\mu$. The coarse grained spacetime elsewhere is specified by a piecewise null stationary initial data hypersurface $N_{-k}[\mu]$ emanating in the $-k^{a}$ direction from $\mu$. It is possible to prescribe initial data on $N_{-k}[\mu]$ so that there is an extremal $X$ surface on it. At this extremal surface, the spacetime is CPT reflected to generate a complete Cauchy slice of $(M[\mu], g[\mu])$.

denoted $(M[\mu], g[\mu])$. Here we include in $(M[\mu], g[\mu])$ both the Cauchy development of the homology slice $H$ and any permissible extension of the Cauchy horizon. This means that $(M[\mu], g[\mu])$ is not necessarily globally hyperbolic, and so some additional work, carried out in the proof of Lemma 1 and Theorem 1, is needed to show that the extremal surface $X$ on $N_{-k}[\mu]$ about which we CPT reflect still is the HRT surface of $\mathscr{I}$ in $(M[\mu], g[\mu])$.

For our purposes, the takeaway from this construction is that for any minimar surface, there exists a spacetime $(M[\mu], g[\mu])$ in which $\mu$ is null-separated along $-k^{a}$ from the HRT surface $X$ of $\mathscr{I}$. Furthermore, since the construction of $(M[\mu], g[\mu])$ makes no assumptions about global hyperbolicity [44], we are free to apply it in the context under consideration in this article.

\section{Apparent horizons lie behind event horizons}

In this section, we argue that minimar surfaces must lie behind event horizons. The proof hinges on the well-known requirement discussed in section 1 that for consistency of the proposal (1.1), the HRT surface of $\mathscr{I}$ must be spacelike- or null-separated to $\mathscr{I}{ }^{10}$ In modifying the area of the HRT surface, we would by the holographic prescription also modify $S_{\mathrm{vN}}\left[\rho_{\mathrm{bdy}}\right]$. Since the latter is invariant under local unitary operations, we immediately arrive at a contradiction; thus HRT surfaces must lie behind event horizons [40].

From this requirement of causal wedge inclusion, we will show that minimar surfaces must also lie behind an event horizon in the original spacetime. To prove this second step, it is critical that strictly classical GR satisfying the null convergence condition admit no evaporating singularities. Let us now make this requirement precise:

Definition 1. An asymptotically AdS spacetime $(M, g)$ is said to be devoid of evaporating singularities if for every closed set $K \subset M$, when $\partial \hat{K}$ is a compact hypersurface in the conformal completion then $\hat{K}$ is compact in the conformal completion.

\footnotetext{
${ }^{10}$ Readers concerned about recent results involving quantum extremal surfaces [46] outside of horizons [49] should defer their concern to section 5 , where we discuss quantum corrections and non-standard boundary conditions.
} 

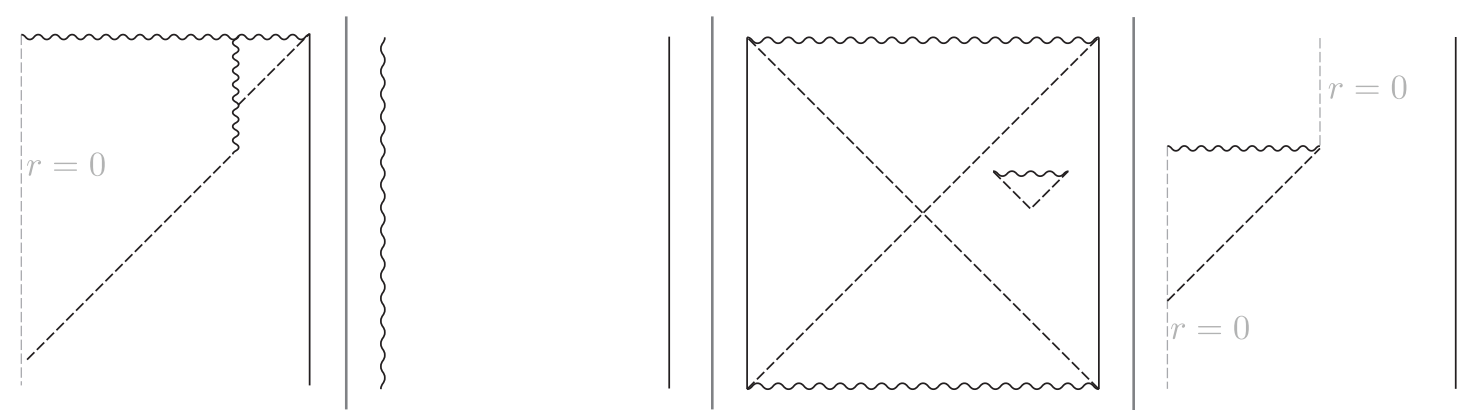

Figure 6. Sketched conformal diagrams of four spacetimes. The two left-most spacetimes are neither globally hyperbolic nor strongly asymptotically predictable, but they are devoid of evaporating singularities. The two right-most spacetimes contain evaporating singularities.

This property, by Lemma 8.2.1 of [26], ensures that no inextendible curves are imprisoned in $\hat{K}$, so that as we follow an inextendible geodesic in $K$ they either leave $K$ through $\partial K$ or go to the conformal boundary. In particular, this rules out geodesic incompleteness between complete hypersurfaces that do not touch singularities. In the special case where $\partial K$ is the union of two achronal surfaces, then compactness of $\hat{K}$ implies global hyperbolicity of $\hat{K}$.

As explained in the introduction, we will assume that all strictly classical solutions to GR that admit a UV completion in quantum gravity are devoid of evaporating singularities. We emphasize that this is a far weaker assumption than strong asymptotic predictability [26], since spacetime can violate global hyperbolicity arbitrarily badly near the asymptotic region owing to non-evaporating singularities. Our assumption further means that no permissible extension of the Cauchy horizon results in an evaporating singularity in classical GR. See figure 6 for some examples.

From this, we prove that the choice of homology hypersurface is immaterial to the definition of $O_{W}[\mu]$ :

Lemma 1. Let $(M, g)$ be devoid of evaporating singularities. If $H_{1}$ and $H_{2}$ are two homology hypersurfaces of a surface $\mu$ homologous to $\mathscr{I}$, then $D\left[H_{1}\right]=D\left[H_{2}\right]$.

Proof. To prove this, it is sufficient to show that every inextendible timelike curve intersecting $H_{1}$ also either intersects $H_{2}$ or reaches the conformal boundary (since $H_{1}$ includes a slice of $\mathscr{I})$. Assume first that $H_{1} \cap H_{2}=\varnothing$, and let $C_{1}$ and $C_{2}$ be the Cauchy slices of $\mathscr{I}$ where they are anchored. By global hyperbolicity and spatial compactness of $\mathscr{I}$ there is a compact subset $I \subset \mathscr{I}$ with $\partial I=C_{1} \cup C_{2}$. Then $\partial \hat{K} \equiv I \cup H_{1} \cup H_{2}$ is a compact hypersurface in the conformal completion (see figure 7 for an illustration), and the region $\hat{K}$ bounded by $\partial \hat{K}$ in the conformal completion is also compact.

Assume an inextendible timelike curve $\gamma$ enters $K$ through $H_{1}$, and assume without loss of generality that it enters $K$ to the future. By compactness of $\hat{K}, \gamma$ must either reach the conformal boundary or leave $K$ to its future. It cannot leave $K$ through $H_{1}$ by global hyperbolicity of $K$. Thus if $\gamma$ does not go to the conformal boundary, it intersects $H_{2}$. 

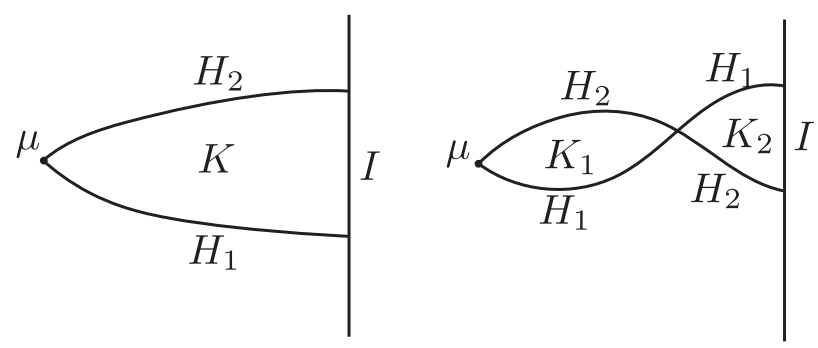

Figure 7. Illustration of two homology slices, which by the lack of evaporating singularities, must have the same outer wedge.

In the case where $H_{1}$ and $H_{2}$ intersect we potentially get multiple compact regions $\hat{K}_{1}, \hat{K}_{2}, \ldots$ bounded by $H_{1} \cup H_{2} \cup I$, as shown in figure 7 . Since the above argument will apply to each region we again find that $\gamma$ intersects $H_{2}$ or reaches $I .{ }^{11}$

For the remaining of the paper, unless stated otherwise we will make our above assumption that classical GR allows no evaporating singularities that satisfy the null convergence condition.

Theorem 1. Let $\mu$ be a future minimar surface in $(M, g)$. Then $\mu$ lies behind the future event horizon in $(M, g)$.

Proof. Let $\Sigma_{\text {tot }}$ be an initial data slice of the spacetime $(M[\mu], g[\mu])$ consisting of $N_{-k}[\mu]$, $H$, and $\widetilde{H} \cdot{ }^{12}$ Within $D\left[\Sigma_{\text {tot }}\right]$, we know that the extremal surface $X[\mu]$ constructed via the above prescription is the minimal area extremal surface in $D\left[\Sigma_{\text {tot }}\right]$. If this remains true for any permissible extension of the Cauchy horizon, then $X[\mu]$ must be the HRT surface of $(M[\mu], g[\mu])$.

Suppose now that there is a permissible extension of the Cauchy horizon containing an extremal surface $X^{\prime}$ also homologous to $\mathscr{I}$. Since the total CFT state on all boundaries is pure, if $X^{\prime}$ were to be the HRT surface of $\mathscr{I}$, then it would also be the HRT surface of the complement $\widetilde{I}$ by complementary recovery of classical holographic codes, and so it would have to be homologous to $\widetilde{I}$. From the $\mathscr{I}$ and $\widetilde{I}$ homology hypersurfaces of $X^{\prime}$ we construct the complete hypersurface $\Sigma_{\text {tot }}^{\prime}$ as the union (see illustration in figure 8). By the absence of evaporating singularities and the requirement that homology hypersurfaces are compact in the conformal completion, the region between $\Sigma_{\text {tot }}$ and $\Sigma_{\text {tot }}^{\prime}$ is globally hyperbolic. But this means that $D\left[\Sigma_{\text {tot }}^{\prime}\right]=D\left[\Sigma_{\text {tot }}\right]$, and so $X^{\prime} \subset D\left[\Sigma_{\text {tot }}\right]$. This contradicts the assumption that $X^{\prime}$ lies in the extension beyond the Cauchy horizon. Thus the proof that $X[\mu]$ is the HRT surface from the case where $(M[\mu], g[\mu])$ is globally hyperbolic still applies, and so $\mu$ must be behind the horizon in $(M[\mu], g[\mu])$.

Consider now the subset $Z=J^{+}\left[\partial^{+} O_{W}[\mu]\right]-J^{+}[\mu]$ of $M[\mu]$, which we can choose so that it agrees with the corresponding subset of $M$ (see figure 9 for an example). If

\footnotetext{
${ }^{11}$ Here we are ignoring potential issues that could arise if the intersection is a dense measure zero set; we assume that the domain of dependence of any one of these hypersurfaces is codimension-zero, and thus that we can "wiggle" the hypersurface to avoid that scenario.

${ }^{12}$ Here $\widetilde{A}$ refers to the CPT conjugate of a quantity $A \in O_{W}[\mu]$ in the coarse-grained spacetime.
} 


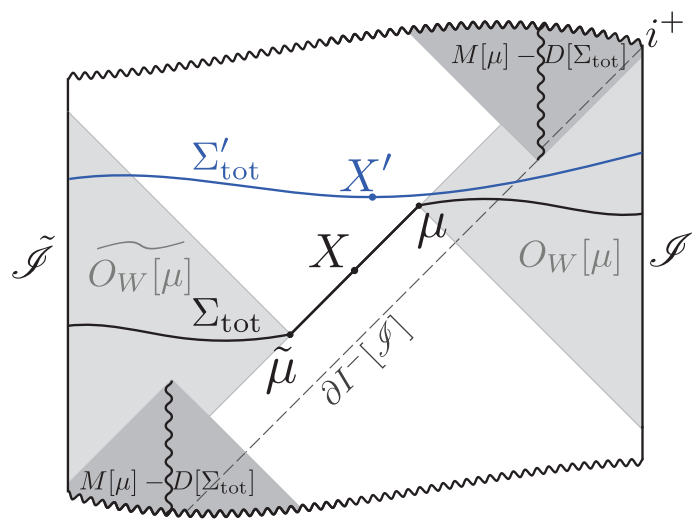

Figure 8. Illustration of a coarse grained spacetime extended beyond Cauchy horizons into the dark gray regions. $X^{\prime}$ is an extremal surface that is a candidate HRT surface. Since $X^{\prime}$ must be homologous to both $\mathscr{I}$ and $\tilde{\mathscr{I}}$, it will lie in $D\left[\Sigma_{\text {tot }}\right] . \mu$ must be spacelike to $\tilde{i}^{+}$for any choice of extension $M[\mu]-D\left[\Sigma_{\text {tot }}\right]$.

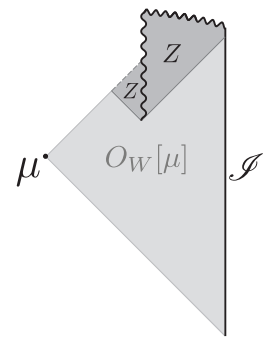

Figure 9. Example of a Cauchy extension $Z$ of the outer wedge that is spacelike to $\mu$. The finegrained spacetime $M$ will induce a particular choice of $Z$. It is possible to choose the coarse grained spacetime so that $O_{W}[\mu] \cup Z \subset M[\mu]$.

$\widetilde{i^{+}} \not \subset Z$, then CFT evolution proceeds beyond $\mathscr{I} \cap Z$, and $D\left[\Sigma_{\text {tot }}\right] \cup Z$ can be extended further. However, a maximal extension of $D\left[\Sigma_{\text {tot }}\right] \cup Z$ must put $\mathscr{I}$ in causal contact with $\mu$, violating causal wedge inclusion, and so we conclude that $\widetilde{i^{+}} \subset Z$. Thus $\mu$ must lie behind the horizon in $O_{W}[\mu] \cup Z$, and by the absence of closed timelike curves this must remain true when completing $O_{W}[\mu] \cup Z$ into $M$.

\section{Trapped surfaces lie behind event horizons}

Having argued that minimar surfaces lie behind event horizons, we proceed to show the main result of this article, that trapped surfaces lie behind event horizons. The idea is simple: prove that between a trapped surface and the asymptotic boundary there always exists at least one minimar surface. We will make use of the following theorem:

Theorem $2([42,50])$. Let $\Sigma$ be a compact spacelike hypersurface with an inner boundary and an outer boundary. Assume that the inner boundary is outer trapped and that the outer boundary is outer untrapped. Then $\Sigma$ contains a smooth stable marginally outer trapped surface $\sigma$. 


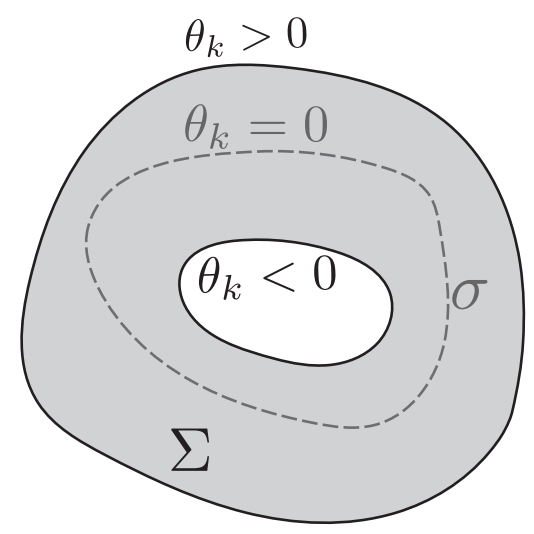

Figure 10. Illustration of a compact spacelike hypersurface $\Sigma$ with an inner boundary that is outer trapped and an outer boundary that is outer untrapped. By Theorem 2 a marginally outer trapped surface $\sigma$ in $\Sigma$ is guaranteed to exist.

The quantities described in this theorem are illustrated in figure 10. In fact, we will use a stronger version of the above theorem that describes how $\sigma$ evolves with an evolving family of spatial slices. We will assume a generic condition on trapped surfaces which is frequently used in classical gravity proofs involved marginally trapped surfaces (see e.g. [51, 52]: $R_{a b} n^{a} n^{b}+\sigma_{a b} \sigma^{a b}>0$, where $\sigma_{a b}$ is the shear tensor and $n^{a}$ is the generator of a null congruence fired orthogonally from the trapped surface. This condition ensures that every leaf in a spacelike hypersurface foliated by marginally outer trapped surfaces is strictly stable [51]. Note that in the following we will only require this condition inside of the outer wedge.

Combining now Theorem 2.1, 3.1 and 6.4 of [53] with the assumption of genericity and its implication of strict stability [51], we have the following: ${ }^{13}$

Theorem 3 ([53]). Let $\Sigma$ be a spacelike hypersurface and let $\partial \Sigma$ consist of two disconnected components $\sigma_{1}$ and $\sigma_{2}$. Assume further that one of these components, $\sigma_{1}$ is outer trapped, while the other $\sigma_{2}$ is outer untrapped. Then the boundary of the outer trapped region on $\Sigma$, denoted $\sigma_{t}$, is (1) a smooth, strictly stable, marginally outer trapped surface homologous to $\sigma_{2}$. Furthermore, if a spacetime region admits a foliation $\left\{\Sigma_{t}\right\}$ by such hypersurfaces, then the union $\mathcal{H}=\bigcup_{t \in[0, T]} \sigma_{t}$ is a piecewise smooth spacelike manifold with a finite number of connected components.

To apply Theorem 3 to prove that trapped surfaces lie behind event horizon, we need $\sigma_{t}$ to also be (1) marginally trapped and (2) homologous to $\mathscr{I}$. To achieve these conditions, we require the implementation of a mild assumption about the past of trapped surfaces $\left(\theta_{\ell}<0, \theta_{k}<0\right)$. Since cosmic censorship is primarily concerned with the future of trapped surfaces, these assumptions do not heavily constrain our results.

\footnotetext{
${ }^{13}$ The theorem is only proven for $D=4$ - we will assume it holds for $D \geq 3$, which appears likely given that Theorem 2 has been proven for $3 \leq D \leq 8$ [50].
} 


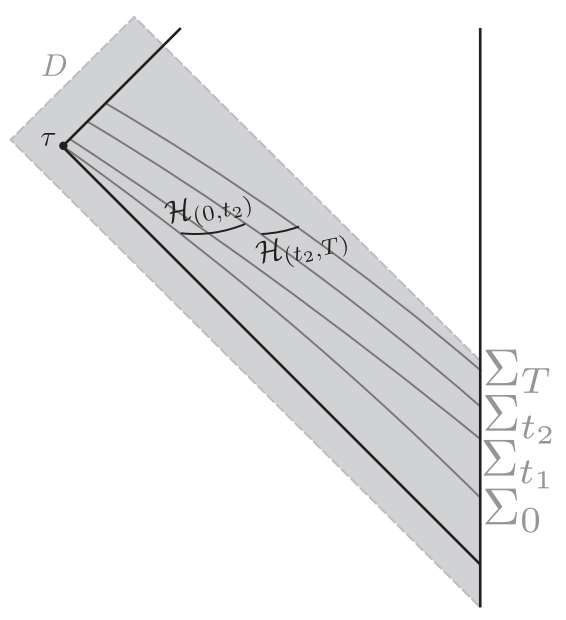

Figure 11. An example of the construction of the manifold $H[\tau]$ outside a past well-behaved trapped surface $\tau$. $\Sigma_{t}$ is a one-parameter family of spatial slices, and both $\mathcal{H}_{\left(0, t_{2}\right)}$ and $\mathcal{H}_{\left(t_{2}, T\right)}$ are spacelike manifolds foliated by marginally outer trapped surfaces. $H[\tau]$ can be taken to be either.

Definition 2. We call a surface $\sigma$ past well-behaved if it is homologous to $\mathscr{I}$ and (1) $C[\sigma]=\partial^{-} O_{W}[\sigma] \cap \mathscr{I}$ is a Cauchy slice of $\mathscr{I}$ and (2) $\partial^{-} O_{W}[\sigma] \subset D$ for some interior of a domain of dependence D. ${ }^{14}$

With this in place we prove a lemma essential for our main result:

Lemma 2. Let $\tau$ be a past well-behaved trapped surface in a generic spacetime $(M, g)$. Then there exists a spacelike manifold $H[\tau]$ in $O_{W}[\tau]$ which is foliated by past well-behaved strictly stable marginally outer trapped surfaces homologous $\mathscr{I}$.

Proof. Pick a smooth one-parameter family of non-intersecting spatial slices $\Sigma_{t}$ for $t \in[0, T]$ that are all contained in $D \cap O_{W}[\tau]$, where $D$ is the interior of the domain of dependence containing $\partial^{-} O_{W}[\tau]$. Pick the hypersurfaces $\Sigma_{t}$ so that they are anchored on Cauchy slices $C_{t}$ of the conformal boundary, and pick the inner boundaries of $\Sigma_{t}$ so they are either sufficiently close to $\tau$ or lying on $N_{k}[\tau]$, so that they are also trapped. By AdS asymptotics and global hyperbolicity in $D$, we can always choose the family so that each $\Sigma_{t}$ has an untrapped surface near $\mathscr{I}$. Theorem 3 now guarantees the existence of a set $\mathcal{H}$ which is the union of smooth spacelike manifolds foliated by smooth strictly stable marginally outer trapped surfaces $\mu_{t}$ homologous to $C_{t}$ (and thus $\mathscr{I}$ ), together with surfaces where $\mathcal{H}$ jumps. See figure 11. Since we choose our foliation so $\mathcal{H} \subset D$, the $\mu_{t}$ are also past well-behaved. Hence, any of the connected components of $\mathcal{H}$, after removing jump surfaces, satisfies all of our claimed properties of $H[\mu]$.

To find our main result, all that is missing is that at least one of the marginally outer trapped surfaces from the previous lemma is also inner trapped. By the past well-behaved assumption, these surfaces cannot be inner untrapped (so-called "anti-normal"). If the

\footnotetext{
${ }^{14}$ This is morally equivalent to demanding the existence of an open set $\mathcal{O}$ around $\sigma$ that can be covered by surfaces that also fulfill requirement (1).
} 
spacetime were spherically symmetric we would now be done: by past well-behavedness we can fire a spherically symmetric past horizon from $\mathscr{I}$ that hits $\mu_{t}$. Choosing $\Sigma_{t}$ (and thus $\mu_{t}$ ) to respect the spherical symmetry, $\mu_{t}$ would be contained in the horizon, and so $\theta_{\ell}\left[\mu_{t}\right]<0$, where strict inequality is guaranteed by our genericity condition. In the absence of spherical symmetry, this becomes harder to prove. We nevertheless find it to be a reasonable assumption for the following two reasons: (1) there is a continuously large amount of freedom in $\mu_{t}$ given that the choice of foliation $\Sigma_{t}$ is arbitrary. (2) By past well-behavedness we can fire a one-parameter family of horizons from the boundary in $D$ and pick each $\Sigma_{t}$ to lie arbitrarily close to a member of this family of horizons. Thus we can construct an $H[\mu]$ where every leaf lies arbitrarily close to a strictly inner trapped surface (strictness follows from genericity). In fact, if we were allowed to use Theorem 3 directly on null foliations with a measure zero set of non-differentiable points, then we could redo the proof of Lemma 2 with $\Sigma_{t}$ chosen to be future horizons, which would yield a proof that $\theta_{\ell}\left[\mu_{t}\right]<0$ as well. With these justifications in mind, we will simply assume that a past-well behaved $\tau$ has at least one choice of $H[\tau]$ containing an inner trapped leaf $\mu_{t}$. In light of the guaranteed existence of $H[\tau]$ by Lemma 2, this can be considered as an addition to the definition of being past well-behaved.

Finally, we can show our main result.

Theorem 4. Let $\tau$ be a trapped surface. If $\tau$ is past well-behaved or has a trapped surface in its outer wedge which is past well behaved, then no future causal curves from $\tau$ can reach $\mathscr{I}$. In particular, $\tau$ lies behind an event horizon.

Proof. By Lemma 2 we know there is a past well-behaved surface $\mu$ in $O_{W}[\tau]$ satisfying the minimar conditions (1)-(3). We now show that for a surface satisfying past wellbehavedness, only properties (1)-(3) of a minimar are needed for the coarse grained spacetime to exist and having the required property that the extremal surface $X$ on $N_{-k}[\mu]$ is the HRT surface of $\mathscr{I}$. That is, the requirement of minimality of $\mu$ on a homology slice can be exchanged for past well-behavedness, and Theorem 1 still applies to $\mu$.

Existence of the coarse grained spacetime is immediate, since that only relies on properties (1)-(3), as shown in [44]. What remains is to show that $X$ is HRT. The crucial piece used for proving this in [44] was that $X$ is contained in a Cauchy surface of the coarse grained spacetime in which it is minimal. We now show that there is such a Cauchy surface $S$ in the coarse grained spacetime even if we do not assume minimality of $\mu$ on a homology slice.

Let $\Sigma$ be the Cauchy surface of the coarse grained spacetime formed by union of the homology slice of $\mu$ with respect to $\mathscr{I}$, its CPT conjugate, and $N_{-k}[\mu]$. Let us for now work in the maximal Cauchy development $D[\Sigma]$, currently refraining from extending possible Cauchy horizons.

By past well-behavedness in the original spacetime, $\partial^{-} O_{W}[\mu]$ is contained in a globally hyperbolic set there, and there are no singularities in a small neighbourhood around it. Since the coarse grained spacetime of $\mu$ shares the same data on $\partial^{-} O_{W}[\mu]$, any potential 


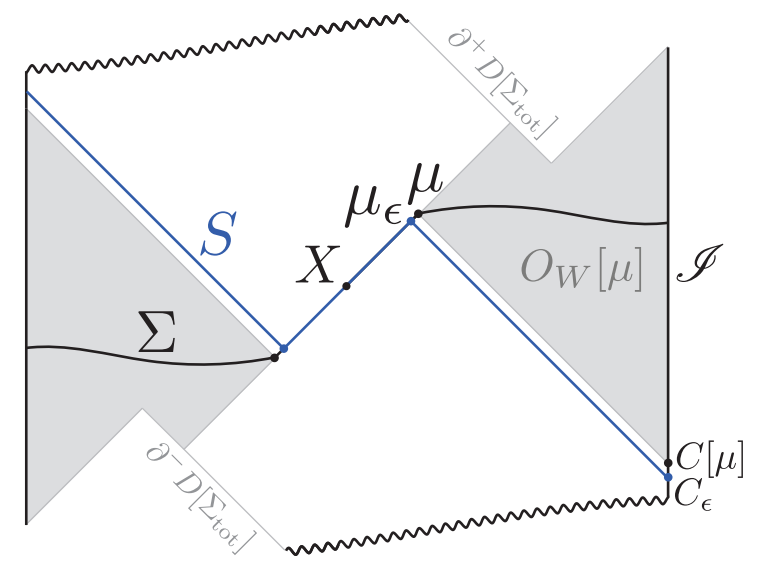

Figure 12. Construction of Cauchy surface $S$ on which $X$ is minimal in the globally hyperbolic region of the coarse grained spacetime $D[\Sigma]$.

singularity in the coarse grained spacetime has to be finitely separated from $\partial^{-} O_{W}[\mu] .{ }^{15}$ Thus, $C[\mu]$ is contained in the interior of $D[\Sigma]$. Generically, $C[\mu]$ will have kinks. Deform $C[\mu]$ slightly to the past into the Cauchy surface $C_{\epsilon}$ to smooth out the kinks, and consider the past horizon $H=\partial I^{+}\left[C_{\epsilon}\right] \cap J^{-}[\Sigma]$ and its intersection with the stationary null congruence, denoted $\mu_{\epsilon}=H \cap N_{-k}[\mu]$. See figure 12. We know that if $\mu_{\epsilon}$ is nonempty, it must lie between $X$ and $\mu$ on $N_{-k}[\mu]$, since $X$ is not causally separated from $\mathscr{I}$. But since $\mu \subset I^{+}\left[C_{\epsilon}\right]$, we know that $\mu_{\epsilon}$ is nonempty and in fact a complete slice of $N_{-k}[\mu]$, since otherwise a generator of $N_{-k}[\mu]$ going from $X$ to $\mu$ would be a causal curve that enters $I^{+}\left[C_{\epsilon}\right]$ without intersecting $\partial I^{+}\left[C_{\epsilon}\right]$.

We now take $S$ to be piecewise null Cauchy slice given by the union of $H, \tilde{H}$ and the part of $N_{-k}[\mu]$ between $\mu_{\epsilon}$ and its CPT conjugate. Since $H$ is a future horizon it has $\theta_{\ell}<0$, and since it ends on $N_{-k}[\mu]$, where every slice has the same area as $\mu$, we get that any surface $\sigma$ contained in $H$ has Area $[\sigma] \geq \operatorname{Area}\left[\mu_{\epsilon}\right]=$ Area $[X]$. Clearly this also holds true for $N_{-k}[\mu]$ and $\tilde{H}$, and thus for the whole of $S$. Hence, we have constructed a Cauchy slice $S$ of $D[\Sigma]$ on which $X$ is minimal. Even though $S$ does not intersect $O_{W}[\mu]$ or $\mu$, the proof from [44] that $X$ is the HRT surface carries through. Furthermore, we are now free to add any permissible extension of the Cauchy horizon, and the absence of evaporating singularities guarantees that $X$ remains that HRT surface, as shown in the proof of Theorem 1. But now Theorem 1 guarantees that $\mu$ lies behind the future event horizon of $\mathscr{I}$, and since $\mu \subset O_{W}[\tau], \tau$ lies behind the event horizon as well.

A simple corollary of our main result follows; we will relax our assumption that classical GR is devoid of evaporating singularities now:

Corollary 1. If there exists a past well behaved trapped surface $\tau$ in a classical asymptotically AdS spacetime $(M, g)$ satisfying the null convergence condition, then at least one of the following holds:

\footnotetext{
${ }^{15}$ We have not ruled out that there is a stress tensor shock along $\partial^{-} O_{W}[\mu]$, but this would not ruin global hyperbolicity.
} 
1. $(M, g)$ has an event horizon, and $\tau$ lies behind it;

2. Classical GR admits solutions with evaporating singularities (in particular, there exist solutions with $O_{W}[\tau]$ and evaporating singularities);

3. $(M, g)$ has no holographic dual.

\section{Discussion}

We have shown from the holographic dictionary that trapped surfaces, hallmarks of strong gravity, must be cloaked from the asymptotic boundary by event horizons. This proof comes at the heels of the holographic derivation of the Penrose Inequality in [33]; both results constitute strong evidence in favor of validity of a version of weak cosmic censorship in gravitational theories whose UV completion is well-described by AdS/CFT, even though the conjecture might be false in gravitational theories without such a UV completion. That is, these derivations suggest that there may be a formulation of weak cosmic censorship that is enforced by quantum gravity in the classical regime (which may not be valid as a statement about gravitational theories that do not exist as classical limits to quantum gravity). It is thus tempting to speculate that the location of trapped surfaces may serve as a "swampland" criterion of sorts [54]. We will not subscribe to such an interpretation here, but rather discuss the precise implications of our results in holography.

Before we do so, however, let us briefly comment on the assumption that evaporating singularities are absent in classical GR. This may seem like an odd assumption to make given that the actual singularity region is not described by the classical theory. However, the absence of evaporating singularities is a constraint on the predictions of the classical theory: regardless of whether we expect quantum corrections to be important in that regime, we may still ask what purely classical gravity would predict. This does not, however, preclude an investigation of the location of quantum trapped surfaces [55] and quantum cosmic censorship in (perturbative) quantum gravity where the null energy condition is violated; that is indeed a natural next step. ${ }^{16}$

Typicality of black holes: the proof that trapped surfaces imply the existence of event horizons pairs nicely with the Penrose singularity theorem [56], which states that trapped surfaces imply the existence of singularities, and so singularities are at least as typical as trapped surfaces. For an asymptotic observer dreaming of immortality, the Penrose singularity theorem can be a foreboding omen. ${ }^{17}$ However, our result should provide some relief to the observer, since it shows that event horizons are just as typical, in the sense that every time the Penrose theorem is invoked to deduce the existence of a singularity in a holographic theory, we can also deduce the existence of an event horizon hiding this singularity. Since a black hole usually is defined by the existence of an event horizon, our result shows that black holes are as typical as trapped surfaces. Black holes always appear

\footnotetext{
${ }^{16} \mathrm{NE}$ thanks R. Bousso and M. Tomasevic for extensive conversations on this.

${ }^{17}$ Indeed, as Wald describes it [7], the formation of naked singularities would be a potential mechanism for a mad scientist to destroy the universe.
} 
whenever gravity gets strong enough to focus both ingoing and outgoing lightrays. This is especially reassuring given the prevalence of gedankenexperiments (in holography and beyond!) whose conclusions are reliant upon the formation of event horizons as a direct consequence of typical gravitational collapse.

Trapped surfaces, horizons, and complexity: in general, knowledge of the spacetime geometry at finite time is insufficient to locate event horizons: the latter are teleological with respect to $\mathscr{I}$. Our result provides a time-local constraint on the existence and location of event horizons from the behavior of trapped surfaces, whose location (and existence) can be ascertained in the neighborhood of a single moment of time. This suggests a potential application to the work starting with [57-59] on the connection between the event horizon and maximal chaos and fast scrambling in holography. Should particular moment-of-time data in the CFT be sufficient to deduce the existence of a trapped surface, this data must be also be sufficient to detect such signatures of event horizons, although the converse is false. This raises an interesting question: if trapped surfaces are a time-local guarantee for the existence of event horizons, what is the CFT dual of a trapped surface?

Some work has already been done in this direction: in [43, 44], it was conjectured that the coarse-grained spacetime associated to an apparent horizon $\mu$ is dual to a CFT quantum state $\rho_{\text {coarse }}$ that preserves all correlators of "simple CFT operators" with simple sources turned on:

$$
\operatorname{Tr}\left[\rho \mathcal{O}_{\text {simple }}\right]=\operatorname{Tr}\left[\rho_{\text {coarse }} \mathcal{O}_{\text {simple }}\right],
$$

where by simple operators we mean CFT operators with support in $\mathscr{I} \cap O_{W}[\mu]$ that result in causal propagation in the bulk, and we have suppressed the sources (and $\rho$ is the original state). On the other hand, the expectation value of highly complex operators is allowed to differ between the two states. Consistent with expectations about complexity of operators localized to the deep black hole interior [60,61], operators localized in the spacetime behind $\mu$ should not be simply reconstructible with access to $\mathscr{I} \cap O_{W}[\mu]$. In particular, the simple entropy proposal, if correct, would imply that correlation functions of a small number of the local CFT primaries of HKLL [62-64] restricted to $O_{W}[\mu] \cap \mathscr{I}$ should not contain the information about the spacetime behind the associated minimar surface $\mu$. Our results serve as an important consistency check to this: if a trapped surface could be in the causal wedge of $\mathscr{I}$, then there would - by Theorem 3 - be a minimar surface in the causal wedge; but then parts of the region behind $\mu$ would be reconstructible from simple operators in $\mathscr{I} \cap O_{W}[\mu]$ by HKLL, in contradiction with the expectation that only complex operators are sensitive to the physics behind $\mu$.

Thus, since trapped surfaces are generally behind minimar surfaces, trapped surfaces appear to be a robust time-local signal of bulk physics that cannot be reconstructed from the CFT with "simple experiments" (under assumption of the simple entropy proposal). In fact, via the simple entropy conjecture, there are protocols that employ simple operators that allow reconstruction of physics outside of minimars but inside horizons (see also upcoming work [65]). We could speculate that minimars (and their associated holographic screens $[52,66])$ are the boundary inside of which simple reconstruction methods break down; since we have shown that these surfaces are not found in the asymptotic region, our 
results are suggestive of a time-local separation between simple and complex physics in the bulk not dissimilar from the one presented in [61]. ${ }^{18}$

This is of course in harmony with the proposed relation between operator CFT complexity and bulk depth in [67-69]. In upcoming work [70], we give a precise covariant description in which regions deeper in the black hole are of higher complexity than their shallower counterparts.

Quantum corrections and black hole evaporation: in purely classical gravity, the assumption of the absence of evaporating singularities is quite reasonable: to our knowledge, known examples of evaporating singularities always involve some violation of the null energy condition. Thus our statement should really be viewed as a prediction of quantum gravity on the behavior of strictly classical GR with matter that is consistent with holography. What about quantum corrections?

Once quantum backreaction is included - in particular, once black holes can evaporate — it is possible for trapped surfaces to lie outside of event horizons. Interestingly, however, this effect appears at later times in the evaporation process (see e.g. [41]). Thus it is tempting to speculate that even in the perturbative quantum gravity regime, at times $t$ much smaller than the Page time, the classical GR result remains valid: trapped surfaces lie behind the event horizon. We emphasize that this is purely speculative: our derivation does not apply in such a setting since the event horizon is teleological and takes into account the entire evaporation process.

Might we expect a quantum version of our statement to be valid? That is, do quantum trapped surfaces [55] lie behind event horizons? In [49], quantum extremal surfaces [46] "outside" of the horizon were found; in this case the existence of non-standard boundary conditions at the AdS boundary were crucial. Interestingly, the quantum focusing conjecture [71] in this case nevertheless enforces the absence of causal communication between the quantum extremal surface and $\mathscr{I}$. These complications illustrate the subtleties that must be accounted for in formulating a quantum version of our proof, in which evaporating singularities can no longer be ignored: the absence of causal communication from quantum trapped surfaces to $\mathscr{I}$ is not equivalent to the absence of quantum trapped surfaces in the causal wedge. This suggests that the correct generalization may actually involve an understanding of whether communication can occur in practice rather than whether or not it is forbidden by causal structure.

\section{Acknowledgments}

It is a pleasure to thank C. Akers, R. Bousso, R. Emparan, S. Fischetti, G. Horowitz, P. Jefferson, S. Leutheusser, and M. Tomasevic for discussions. This work is supported in part by NSF grant no. PHY-2011905 and the MIT department of physics. The work of NE is also supported in part by the U.S. Department of Energy, Office of Science, Office

\footnotetext{
${ }^{18}$ The reader may prima facie suspect a contradiction here: our results apply to the nearest marginally trapped surface, whereas the Python's lunch conjecture applies to the nearest extremal surface. The difference lies in having access to the entire boundary as opposed to just $O_{W}[\mu] \cap \mathscr{I}$. This tension will be discussed more explicitly in [65].
} 
of High Energy Physics of U.S. Department of Energy under grant Contract Number DESC0012567 (High Energy Theory research). The work of ÅF is also supported in part by an Aker Scholarship.

Open Access. This article is distributed under the terms of the Creative Commons Attribution License (CC-BY 4.0), which permits any use, distribution and reproduction in any medium, provided the original author(s) and source are credited.

\section{References}

[1] S.W. Hawking, Gravitational radiation from colliding black holes, Phys. Rev. Lett. 26 (1971) 1344 [INSPIRE].

[2] J.D. Bekenstein, Black holes and the second law, Nuovo Cim. Lett. 4 (1972) 737.

[3] J.M. Bardeen, B. Carter and S.W. Hawking, The four laws of black hole mechanics, Commun. Math. Phys. 31 (1973) 161 [InSPIRE].

[4] J.L. Friedman, K. Schleich and D.M. Witt, Topological censorship, Phys. Rev. Lett. 71 (1993) 1486 [Erratum ibid. 75 (1995) 1872] [gr-qc/9305017] [INSPIRE].

[5] R. Penrose, Gravitational collapse: the role of general relativity, Riv. Nuovo Cim. 1 (1969) 252 [Gen. Rel. Grav. 34 (2002) 1141].

[6] R.P. Geroch and G.T. Horowitz, Global structure of spacetimes, in General relativity: an Einstein centenary survey, S. Hawking and W. Israel eds., Cambridge University Press, Cambridge U.K. (1979).

[7] R.M. Wald, Gravitational collapse and cosmic censorship, 10, 1997, DOI [gr-qc/9710068] [INSPIRE].

[8] J. Santos, Connecting the weak gravity conjecture to the weak cosmic censorship, talk given at the Strings 2018, June 25-29, Okinawa, Japan (2018).

[9] G.T. Horowitz, J.E. Santos and B. Way, Evidence for an electrifying violation of cosmic censorship, Class. Quant. Grav. 33 (2016) 195007 [arXiv: 1604.06465] [INSPIRE].

[10] T. Crisford and J.E. Santos, Violating the weak cosmic censorship conjecture in four-dimensional Anti-de Sitter space, Phys. Rev. Lett. 118 (2017) 181101 [arXiv: 1702.05490] [INSPIRE].

[11] T. Crisford, G.T. Horowitz and J.E. Santos, Testing the weak gravity - Cosmic censorship connection, Phys. Rev. D 97 (2018) 066005 [arXiv: 1709.07880] [INSPIRE].

[12] T. Crisford, G.T. Horowitz and J.E. Santos, Attempts at vacuum counterexamples to cosmic censorship in AdS, JHEP 02 (2019) 092 [arXiv:1805.06469] [INSPIRE].

[13] G.T. Horowitz and J.E. Santos, Further evidence for the weak gravity - Cosmic censorship connection, JHEP 06 (2019) 122 [arXiv:1901.11096] [INSPIRE].

[14] R. Gregory and R. Laflamme, Black strings and p-branes are unstable, Phys. Rev. Lett. 70 (1993) 2837 [hep-th/9301052] [INSPIRE].

[15] R. Gregory and R. Laflamme, The instability of charged black strings and p-branes, Nucl. Phys. B 428 (1994) 399 [hep-th/9404071] [InSPIRE].

[16] L. Lehner and F. Pretorius, Black strings, low viscosity fluids, and violation of cosmic censorship, Phys. Rev. Lett. 105 (2010) 101102 [arXiv:1006.5960] [INSPIRE]. 
[17] P. Figueras, M. Kunesch and S. Tunyasuvunakool, End point of black ring instabilities and the weak cosmic censorship conjecture, Phys. Rev. Lett. 116 (2016) 071102 [arXiv: 1512.04532] [INSPIRE].

[18] P. Figueras, M. Kunesch, L. Lehner and S. Tunyasuvunakool, End point of the ultraspinning instability and violation of cosmic censorship, Phys. Rev. Lett. 118 (2017) 151103 [arXiv: 1702.01755] [INSPIRE].

[19] T. Andrade, R. Emparan, D. Licht and R. Luna, Cosmic censorship violation in black hole collisions in higher dimensions, JHEP 04 (2019) 121 [arXiv:1812.05017] [INSPIRE].

[20] T. Andrade, R. Emparan, D. Licht and R. Luna, Black hole collisions, instabilities, and cosmic censorship violation at large D, JHEP 09 (2019) 099 [arXiv: 1908.03424] [INSPIRE].

[21] T. Andrade, P. Figueras and U. Sperhake, Violations of weak cosmic censorship in black hole collisions, arXiv:2011.03049 [INSPIRE].

[22] M.W. Choptuik, Universality and scaling in gravitational collapse of a massless scalar field, Phys. Rev. Lett. 70 (1993) 9 [INSPIRE].

[23] D. Christodoulou, Examples of naked singularity formation in the gravitational collapse of a scalar field, Annals Math. 140 (1994) 607 [INSPIRE].

[24] R.S. Hamade and J.M. Stewart, The spherically symmetric collapse of a massless scalar field, Class. Quant. Grav. 13 (1996) 497 [gr-qc/9506044] [INSPIRE].

[25] S.W. Hawking and G.F.R. Ellis, The large scale stucture of space-time, Cambridge University Press, Cambridge U.K. (1973).

[26] R.M. Wald, General relativity, The University of Chicago Press, Chicago U.S.A. (1984).

[27] N. Arkani-Hamed, L. Motl, A. Nicolis and C. Vafa, The string landscape, black holes and gravity as the weakest force, JHEP 06 (2007) 060 [hep-th/0601001] [INSPIRE].

[28] F.J. Tipler, Singularities in conformally flat spacetimes, Phys. Lett. A 64 (1977) 8 [INSPIRE].

[29] C.J.S. Clarke, Naked singularities and causality violation, in Relativistic astrophysics and cosmology, V. de Sabbata and T.M. Karade eds., Springer, Germany (1984).

[30] B.C. Nolan, Strengths of singularities in spherical symmetry, Phys. Rev. D 60 (1999) 024014 [gr-qc/9902021] [INSPIRE].

[31] A. Ori, Strength of curvature singularities, Phys. Rev. D 61 (2000) 064016 [InSPIRE].

[32] R. Emparan, Predictivity lost, predictivity regained: a Miltonian cosmic censorship conjecture, Int. J. Mod. Phys. D 29 (2020) 2043021 [arXiv:2005.07389] [inSPIRE].

[33] N. Engelhardt and G.T. Horowitz, Holographic argument for the Penrose inequality in AdS spacetimes, Phys. Rev. D 99 (2019) 126009 [arXiv:1903.00555] [InSPIRE].

[34] R. Penrose, Naked singularities, Ann. New York Acad. Sci. 224 (1973) 125.

[35] I. Itkin and Y. Oz, Penrose inequality for asymptotically AdS spaces, Phys. Lett. B 708 (2012) 307 [arXiv: 1106. 2683] [INSPIRE].

[36] V. Husain and S. Singh, Penrose inequality in Anti-de Sitter space, Phys. Rev. D 96 (2017) 104055 [arXiv: 1709.02395] [INSPIRE].

[37] S. Ryu and T. Takayanagi, Holographic derivation of entanglement entropy from AdS/CFT, Phys. Rev. Lett. 96 (2006) 181602 [hep-th/0603001] [INSPIRE]. 
[38] V.E. Hubeny, M. Rangamani and T. Takayanagi, A covariant holographic entanglement entropy proposal, JHEP 07 (2007) 062 [arXiv:0705.0016] [INSPIRE].

[39] A.C. Wall, Maximin surfaces, and the strong subadditivity of the covariant holographic entanglement entropy, Class. Quant. Grav. 31 (2014) 225007 [arXiv:1211.3494] [INSPIRE].

[40] M. Headrick, V.E. Hubeny, A. Lawrence and M. Rangamani, Causality \& holographic entanglement entropy, JHEP 12 (2014) 162 [arXiv:1408.6300] [INSPIRE].

[41] R. Bousso and N. Engelhardt, Generalized second law for cosmology, Phys. Rev. D 93 (2016) 024025 [arXiv: 1510.02099] [INSPIRE].

[42] L. Andersson and J. Metzger, The area of horizons and the trapped region, Commun. Math. Phys. 290 (2009) 941 [arXiv: 0708.4252] [INSPIRE].

[43] N. Engelhardt and A.C. Wall, Decoding the apparent horizon: coarse-grained holographic entropy, Phys. Rev. Lett. 121 (2018) 211301 [arXiv:1706.02038] [INSPIRE].

[44] N. Engelhardt and A.C. Wall, Coarse graining holographic black holes, JHEP 05 (2019) 160 [arXiv: 1806.01281] [INSPIRE].

[45] C. Akers, J. Koeller, S. Leichenauer and A. Levine, Geometric constraints from subregion duality beyond the classical regime, arXiv:1610.08968 [INSPIRE].

[46] N. Engelhardt and A.C. Wall, Quantum extremal surfaces: holographic entanglement entropy beyond the classical regime, JHEP 01 (2015) 073 [arXiv:1408.3203] [INSPIRE].

[47] P.M. Chesler and B. Way, Holographic signatures of critical collapse, Phys. Rev. Lett. 122 (2019) 231101 [arXiv:1902.07218] [INSPIRE].

[48] T. Hertog and G.T. Horowitz, Holographic description of AdS cosmologies, JHEP 04 (2005) 005 [hep-th/0503071] [INSPIRE].

[49] A. Almheiri, R. Mahajan and J. Maldacena, Islands outside the horizon, arXiv:1910.11077 [INSPIRE].

[50] L. Andersson, M. Eichmair and J. Metzger, Jang's equation and its applications to marginally trapped surfaces, arXiv:1006.4601 [INSPIRE].

[51] A. Ashtekar and B. Krishnan, Dynamical horizons and their properties, Phys. Rev. D 68 (2003) 104030 [gr-qc/0308033] [InSPIRE].

[52] R. Bousso and N. Engelhardt, Proof of a new area law in general relativity, Phys. Rev. D 92 (2015) 044031 [arXiv: 1504.07660] [INSPIRE].

[53] L. Andersson, M. Mars, J. Metzger and W. Simon, The time evolution of marginally trapped surfaces, Class. Quant. Grav. 26 (2009) 085018 [arXiv:0811.4721] [INSPIRE].

[54] C. Vafa, The string landscape and the swampland, hep-th/0509212 [INSPIRE].

[55] A.C. Wall, The generalized second law implies a quantum singularity theorem, Class. Quant. Grav. 30 (2013) 165003 [Erratum ibid. 30 (2013) 199501] [arXiv:1010.5513] [INSPIRE].

[56] R. Penrose, Gravitational collapse and space-time singularities, Phys. Rev. Lett. 14 (1965) 57 [INSPIRE].

[57] S.H. Shenker and D. Stanford, Black holes and the butterfly effect, JHEP 03 (2014) 067 [arXiv: 1306.0622] [INSPIRE].

[58] D.A. Roberts, D. Stanford and L. Susskind, Localized shocks, JHEP 03 (2015) 051 [arXiv: 1409.8180] [INSPIRE]. 
[59] J. Maldacena, S.H. Shenker and D. Stanford, A bound on chaos, JHEP 08 (2016) 106 [arXiv: 1503.01409] [INSPIRE].

[60] D. Harlow and P. Hayden, Quantum computation vs. firewalls, JHEP 06 (2013) 085 [arXiv: 1301.4504] [INSPIRE].

[61] A.R. Brown, H. Gharibyan, G. Penington and L. Susskind, The Python's Lunch: geometric obstructions to decoding Hawking radiation, JHEP 08 (2020) 121 [arXiv: 1912.00228] [INSPIRE].

[62] A. Hamilton, D.N. Kabat, G. Lifschytz and D.A. Lowe, Local bulk operators in AdS/CFT: a boundary view of horizons and locality, Phys. Rev. D 73 (2006) 086003 [hep-th/0506118] [INSPIRE].

[63] A. Hamilton, D.N. Kabat, G. Lifschytz and D.A. Lowe, Holographic representation of local bulk operators, Phys. Rev. D 74 (2006) 066009 [hep-th/0606141] [INSPIRE].

[64] A. Hamilton, D.N. Kabat, G. Lifschytz and D.A. Lowe, Local bulk operators in AdS/CFT: a holographic description of the black hole interior, Phys. Rev. D 75 (2007) 106001 [Erratum ibid. 75 (2007) 129902] [hep-th/0612053] [INSPIRE].

[65] N. Engelhardt, G. Penington and A. Shahbazi-Moghaddam, Life without pythons would be so simple, to appear.

[66] R. Bousso and N. Engelhardt, New area law in general relativity, Phys. Rev. Lett. 115 (2015) 081301 [arXiv: 1504.07627] [INSPIRE].

[67] L. Susskind, Why do things fall?, arXiv:1802.01198 [INSPIRE].

[68] L. Susskind, Complexity and Newton's laws, Front. in Phys. 8 (2020) 262 [arXiv: 1904.12819] [INSPIRE].

[69] L. Susskind and Y. Zhao, Complexity and momentum, JHEP 21 (2020) 239 [arXiv:2006.03019] [INSPIRE].

[70] N. Engelhardt and Å. Folkestad, to appear.

[71] R. Bousso, Z. Fisher, S. Leichenauer and A.C. Wall, Quantum focusing conjecture, Phys. Rev. D 93 (2016) 064044 [arXiv: 1506.02669] [INSPIRE]. 